\title{
Differences in Socio-demographics Status, Risk Behaviours, Healthcare Uptake and HIV/STIs Between Brothel-based and Street-based Female Sex Workers in Yunnan, China
}

\section{Guoxi Cai}

Nagasaki University: Nagasaki Daigaku

Yufen Liu

Centers for Disease Control

Jinman Zhuang

Fujian Medical University

Yixiao Lu

Nagasaki University: Nagasaki Daigaku

Jiwen Wu

Nagasaki University: Nagasaki Daigaku

Zhijian Hu

Fujian Medical University

Jianping Zhang

Kunming Medical University

Fei He ( $\nabla$ i.fei.he@fjmu.edu.cn )

Fujian Medical University

\section{Research Article}

Keywords: China, HIV infection, STI prevalence, epidemiology, sex workers, behaviours, healthcare services

Posted Date: May 25th, 2021

DOI: https://doi.org/10.21203/rs.3.rs-529460/v1

License: (c) (1) This work is licensed under a Creative Commons Attribution 4.0 International License. Read Full License 


\section{Differences in socio-demographics status, risk behaviours, healthcare uptake and HIV/STIs between brothel-based and street-based female sex workers in Yunnan, China}

Guoxi Cai ${ }_{a, b, c}^{\#}$, Yufen Liü ${ }_{d}^{\#}$, Jinman Zhuange, Yixiao Lub, Jiwen Wub,f, Zhijian $\mathrm{Hu}_{\mathrm{e}}$, Jianping Zhang, Fei He* ${ }_{\mathrm{e}}$

a. Department of International Health, Institute of Tropical Medicine (NEKKEN), Nagasaki University, Nagasaki, 852-8523, Japan

b. Department of Public Health, Nagasaki University Graduate School of Biomedical Sciences, Nagasaki, 852-8523, Japan

c. Department of Public Health, Nagasaki Prefectural Institute of Environment and Public Health, Nagasaki, 2-1306-11, Japan

d. Department of Policy and Integration Department, National Centre for AIDS/STD Control and Prevention, China CDC, Beijing, 102206, China

e. Department of Epidemiology and Health Statistics, School of Public Health, Fujian Medical University, Fuzhou, 350108, China; Fujian Provincial Key Laboratory of Environment factors and Cancer; Key Laboratory of Ministry of Education for Gastrointestinal Cancer; Fujian Medical University, Fuzhou, 350108, China; Fujian Digital Institute of Tumor Big Data, Fujian Medical University, Fuzhou 350122, China

f. Department of Human Anatomy and Histoembryology, School of basic medical sciences, Fujian Medical University, Fuzhou, 350108, China

g. Department of the Child-adolescent and Maternal Care of faculty of Public Health, Kunming Medical University, Yunnan 650500, China 
${ }^{\#}$ Guoxi Cai and Yufen Liu contributed equally to this work

* Corresponding author: Department of Epidemiology and Health Statistics, Fujian Provincial Key Laboratory of Environment Factors and Cancer, School of Public Health, Fujian Medical University, Fuzhou 350122, Fujian Province, China E-mail address: i.fei.he@fjmu.edu.cn (Fei He)

Telephone and fax numbers: +8659122862023 


\section{Abstract}

2 Background: Heterosexual contact is the primary mode of HIV transmission in China

3 and commercial sex is thought to play a crucial role in China's epidemic. Female sex

4 works (FSWs) in China tend to be either brothel-based (BSWs) or street-based (SSWs),

5 but few studies have investigated the differences between these important segments of

6 this difficult-to-reach, high-risk population. Our aim was to explore these differences.

7 Methods: A cross-sectional survey was conducted in Yunnan Province of China in

8 partnership with a local FSW-friendly non-governmental organization. Face-to-face

9 interviews using a structured questionnaire were conducted to collect data on

10 socio-demographic characteristics, sex work history, sexual behaviours,

11 HIV/STI-related knowledge, HIV testing history, and healthcare services uptake. Blood

12 samples were taken for HIV and syphilis testing, and urine samples for gonorrhea and

13 chlamydia testing. Descriptive statistics were used to evaluate differences between

14 SSWs and BSWs.

15 Results: A total of 185 BSWs and 129 SSWs were included in the study. SSWs were

16 older and less educated, had more dependents and more clients, accessed fewer

17 healthcare services, and had higher prevalence of HIV/STIs. SSWs also had similar but

18 higher rates of consistent condom use with clients. 
19 Conclusions: Our study provides evidence that confirms the disproportionately high

20 vulnerability of SSWs to HIV and other STIs, underscoring the urgent need for the

21 Chinese health and public health sectors to prioritize outreach to SSWs. Awareness and

22 educational programs, condom distribution, testing and health check-ups should be

23 included in a comprehensive strategy for HIV/STI prevention in this high-risk

24 population.

25 Keywords: China, HIV infection, STI prevalence, epidemiology, sex workers,

behaviours, healthcare services

27

28

29

30 


\section{Background}

Even though China has made significant progress in HIV/AIDS control and

prevention, the epidemic is a continuing challenge (1). Sexual transmission is now the primary driver of China's HIV epidemic, accounting for more than $90 \%$ of new HIV nationwide (2). Although the epidemic is largely characterized by a low national prevalence of $0.037 \%$ (3), HIV prevalence among sex workers overall was $0.2 \%$ in 2019(4), with certain segments of female sex workers (FSWs) having disproportionately higher prevalence (e.g., low-fee FSW at 4.7\%) (5). The number of FSWs in China has been estimated to be between 2 and 20 million (6-8). However, it is a heterogeneous population, and Chinese FSW are often segmented by semi-overlapping work venue or fee level groups $(5,9,10)$. Two such segments are the brothel-based sex workers bars, hotels, guesthouses, saunas, massage parlors, etc.) and street-based sex workers (SSWs), who solicit clients outside, on streets or in parks. While some studies have evaluated socio-demographic characteristics, HIV-related knowledge, risk behaviors, and prevalence of HIV/STIs among BSWs $(9,11-13)$, few studies have investigated the SSWs in China (5, 13-15) - their high mobility and intense secrecy because of fear of arrest and detention makes finding and engaging them in research very challenging (10). 
54 Just three studies, to these authors' knowledge, have focused on comparisons of BSWs and SSWs (16-18). Yunnan Province has one of the highest HIV prevalence in China. Linxiang

57 District is one of the epidemic centers, with a population of 303,600 . With a population 58 of $>300$ thousand, Linxiang borders Myanmar, near Southeast Asia's Golden Triangle region, well known for opium production and trafficking. Although injecting drug use

60 remains a significant mode of HIV transmission in the area, new cases acquired by

61 heterosexual transmission increased from 13\% in 2004 to $60 \%$ in 2008 and sex work is

62 prevalent, fueling the concern for bridging HIV to the general population. Secondly,

63 Linxiang District has well-established grassroots non-governmental organizations

64 (NGOs) that have a long history of working among local FSWs and an active

65 partnership with the local CDC, which has been actively engaged in providing

66 healthcare to the FSWs in the region for many years. Therefore, we conducted a study of BSWs and SSWs in Linxiang District, Yunnan Province, China, comparing their socio-demographic characteristics, sexual behaviours

69 and high-risk practices, experience of verbal or physical abuse, HIV/STI-related knowledge, HIV/STI prevalence, and experience with receiving healthcare. 


\subsection{Study design}

To evaluate differences between BSW and SSW we conducted a cross-sectional

survey between August and September 2008 in Linxiang District Yunnan, China. This study received approval from the Yunnan Center for Disease Control and Prevention (CDC) Institutional Review Board.

\subsection{Study site and field management} educators who worked for one of the Linxiang NGOs were recruited to the study team.

81 They helped in the mapping the two FSW groups, recruiting FSW to participate in the study (including collection of informed consent), and interviewing participants using a

83 structured questionnaire. Blood and urine samples were collected by laboratory staff

84 from the local CDC for HIV/STI testing. HIV/STI-related pre-test and post-test counselling was provided to all participants. Any participant found to have HIV/STIs were referred to free HIV and STI treatment as indicated.

\subsection{Mapping and sampling}


establishments that functioned as brothels and all locations at which the SSWs were

90 known to solicited clients were mapped out before the survey in this study. Based on

91 this work, the study team knew that there were 387 BSWs operating in 53

92 establishments within four geographical blocks in the district and 148 SSWs roaming

93 the streets in the north-western part of the district. the 21 massage salons in these blocks were recruitment sites. To avoid inferring during their working time, we conducted the interviews. For SSWs, time-location sampling

97 (used to collect data from hard-to-reach populations and snowball sampling were adopted. To protect participant privacy, we rented a house in the district so that the

99 interviews, which were conducted by NGO and CDC staff members, could be conducted in private. Each participant was provided 20 CNY (3.5 USD) and free medical service (ie, counselling, testing, and treatment as needed) as an incentive to participate in the study.

\subsection{Data collection}


supplementary files). This instrument was used to collect data on socio-demographic

characteristics, sex worker history, sex behaviours and risky practices, HIV/STI-related

knowledge, risk perception, healthcare service uptake, HIV testing, and self-reported measured by seven true/false/unknown questions. One point was given for each correct answer, with the total score ranging from 0 to 7 points. Knowledge about symptoms of

112 other STIs was measured by 10 questions, with the score ranging from 0 to 10 points.

113 Both focus group discussions and in-depth interviews with FSWs and peer educators

114 were conducted to check the appropriateness of the questionnaire prior to its use in this 115 study.

\subsection{Laboratory testing}

Blood samples were tested for HIV and syphilis. HIV was tested using an enzyme-linked immunosorbent assay (ELISA) for screening and the Western blot test

119 for confirmation. Screening for syphilis was performed using a traditional algorithm.

120 FSW were first screened using a rapid plasma reagin (RPR) test and if positive, a

121 Treponema pallidum particle agglutination (TPPA) test was performed to confirm 
syphilis. Those FSW with positive RPR and TPPA results were considered to probable active syphilis.

Urine samples were tested for gonorrhoea and chlamydia by real-time fluorescent quantitative polymerase chain reaction (FQ-PCR) assay.

\subsection{Variable definition}

The comparison was conducted between the two groups BSWs and SSWs.

Outcome variables were HIV/STIs-related risks, history of sex work, HIV/STIs healthcare service, knowledge, and risk perception.

\subsection{Statistical methods}

Means and proportions were compared (BSWs vs. SSWs) using the t-test, Mann-Whitney U-test, and chi-square test; $P<0.05$ was considered statistically significant (two-sided). All analyses were performed using Statistical Package for the Social Sciences (SPSS) software (v11.5; IBM SPSS Co., USA).

\section{Results}

\subsection{Sociodemographic characteristics and sex worker history}

A total of 185 of a possible 196 (94.4\%) BSWs and 129 of a possible 148 (87.2\%)

SSWs were recruited to the study. The sociodemographic characteristics of BSW and 
SSW participants are shown in Table 1. SSWs were older and more of them were married; they had received less education and had to support more dependents than BSWs $(P<0.001)$. They had begun sex work at an older age, had longer sex worker careers $(P<0.01)$, and received a lower fee per client than BSWs $(P<0.001)$. They also solicited many more clients per day $(P<0.001)$ than their BSW counterparts.

\subsection{Sex behaviours and risky practices}

Risky practices and condom usage are described in Table 2. Drug use within the past year and experience of verbal or physical abuse from clients were reported in both groups (drug use: SSWs vs. BSWs $=6.1 \%$ vs. $4.5 \%, P=0.703$; experience of verbal or physical abuse from clients: SSWs vs. BSWs $=35.4 \%$ vs. $45.5 \%, P=0.078)$. Condoms were the most used contraception method in both groups (SSWs vs. BSWs $=87.3 \%$ vs. $89.1 \%, P=0.623$ ), and $8.1 \%$ of SSWs and $19.2 \%$ of BSWs had experienced condom breakage or slippage during the previous week $(P<0.01)$. The proportion of consistent condom use with clients during the previous week was higher among BSWs than among SSWs (SSWs vs. BSWs $=92.0 \%$ vs. 98.9.0\%, $P<0.01$ ). Conversely, condom use with a boyfriend or husband was higher in SSWs than in BSWs. (condom use with a boyfriend: SSWs vs. BSWs $=42.1 \%$ vs. $31.8 \%, P<0.05$; condom use with husband: SSWs vs. $\mathrm{BSWs}=52.8$ vs. $11.5 \%, P<0.001)$. 
significant difference was found between the two groups (low grade knowledge score of

STIs symptoms: SSWs vs. BSWs $=70.8$ vs. $36.5 \%, P<0.001)$. Compared with BSWs,

SSWs had received fewer HIV/STI related healthcare (ever receive any AIDS-related health service: SSWs vs. BSWs $=37.0 \%$ vs. $64.4 \%, P<0.001)$. About two-thirds of the subjects in both groups had had HIV testing in the past lifetime (SSWs vs. BSWs = $68.5 \%$ vs. $65.5 \%, P=0.588)$ (Table 3 ). symptoms during the past year $(P=0.394)$ (i.e., abnormal vaginal discharge, $21.4 \%$ vs. $24.3 \%, P=0.581$; genital ulcers, $2.4 \%$ vs. $5.1 \%, P=0.234$; dysuria, $15.9 \%$ vs. $15.8 \%$, $P=0.990 ;$ genital itch, $16.8 \%$ vs. $29.3 \%, P<0.05)$. The perception of risk for acquiring HIV/STIs from clients and of transmitting HIV/STIs to clients were both higher among

SSWs than among BSWs (perception of risk for acquiring HIV/STIs from clients: $3)$. 


\subsection{HIV/STIs prevalence}

Five of the SSWs and zero BSWs have HIV infection $(P<0.01)$. Syphilis

177 prevalence was higher in SSWs (SSWs vs. BSWs $=7.0 \%$ vs. $1.1 \%, P<0.01$ ). Overall, $37.2 \%$ of SSWs and $24.9 \%$ of BSWs were found to have HIV/STI infection (Table $4, P$ $<0.05)$.

\section{Discussion}

The main finding of this study was notable differences in characteristics,

behaviours, and sexual health between SSWs and BSWs. To summarize, SSWs tended

to be older, married, less educated, and less engaged with healthcare services. They had

been engaged in sex work for a longer period, solicited more clients each day, and

charged clients lower fees. Although other risk behaviours, condom use with clients,

HIV knowledge, and HIV testing were similar between the two groups, SSWs had

higher rates of both HIV and STIs.

vulnerable groups in society as they must constantly grapple with inequities, power

imbalances, stigma and discrimination, violence, mental health challenges, substance 
assessment. The women who were SSWs in our study were older and less educated,

which, in Chinese society, could mean they were more vulnerable to suffer layoffs and more likely to struggle to find work. Many in this demographic who find themselves out of work turn to sex work to support their families.

Indeed, the results we report herein largely support and confirm the existing literature on SSW. For example, a small 2008 qualitative study by Zeng et al. among eight SSWs found that they were mostly low socio-economic status rural-to-urban migrants with low HIV awareness and prevention knowledge. Major barriers to health services uptake included stigma, fear of arrest, lack of family support, and poor financial means (13). Zhou et al. found that in a 2012 - 2013 study among 781 low-fee FSW, HIV prevalence was $4.7 \%$ and syphilis prevalence was $15.0 \%$. Most were older, married, and less educated, and solicited clients on the street, in self-rented rooms, or in "market day" buildings, meaning that they did not work in established venues (5). In a 2006 - 2007 study comparing SSW and BSW, SSW had lower educational attainment, greater responsibility for supporting family, lower fees for services, longer histories of sex work, more risk behaviours, and higher prevalence of STIs (16). Two other studies found higher rates of syphilis among SSWs compared to other groups of FSWs $(17,18)$. 
211 BSWs and SSWs in our study compared to the literature $(5,11,12,16)$. Although SSWs

212 in our study did report lower consistent condom use that was statistically significant

213 (92\% vs. $99 \%$ among BSW, $P<0.01)$, the difference is small and may not be

214 meaningful. These data do suggest, however, that prevention interventions among FSW

215 in this setting may be making a positive impact and perhaps condom use in the

216 commercial sex setting may be becoming more normalized among FSW and their

217 clients. Nevertheless, there is room for further improvement-both BSW and SSW

218 reported recent issues with condom breakage or slippage, and both also reported

219 dramatically lower rates of condom use with boyfriends and husbands.

Because of severe stigma and discrimination, mistrust of the government, and fear of arrest and incarceration, FSWs in China are a highly mobile and hidden segment of society, especially the subgroup that is SSWs. This makes them difficult to reach with

223 health and public health interventions and with research such as this (10). Our experience in partnering for this research with local grassroots NGO resources suggested that the involvement of such FSW-friendly NGOs and their trained and experienced peer educators is important. Many NGOs have been embedded in their communities for a long time, have taken the care to build trust and be a force for good, 
and many are helping people deal with sensitive healthcare issues and stigmatized or

even criminalized behaviors. Thus, cooperation between government sectors at all levels

230 and NGOs working in the community level in China should be encouraged in order to

231 improve HIV/STI prevention and care across all high-risk groups $(21,22)$. years ago, the continued rise in HIV transmission via heterosexual contact means that

234 FSW remain an important high-risk group in China's HIV epidemic even today $(2,20)$.

235 Secondly, while we did use sampling techniques to recruit participants, which may have

236 introduced some bias, no participants discontinued during the study and therefore our

237 results did not suffer from non-response bias. Thirdly, although there was some potential for social-desirability bias due to the sensitive nature of some of the interview questions, this risk was minimized by ensuring the interviews were conducted by FSWs who were affiliated with FSW-friendly NGOs. Fourthly, it is possible that our results on

241 HIV prevalence reflect an inability of SSWs to work in venues (i.e., to get work as

242 BSWs) because many may disallow HIV-positive status and/or the increased likelihood 243 of HIV-positive status among older FSWs who tended to be SSWs. Our work did not account for such a potential confounding factor. Finally, our small sample size and 
245 cross-sectional design limited the generalizability of our results and precluded any

246 evaluation of causation.

247 5. Conclusion

248 In summary, the differences we observed between BSWs and SSWs in our study

249 were significant and confirm the disproportionately high vulnerability of SSWs to HIV

250 and other STIs. This evidence underscores the urgent need for the Chinese health and

251 public health sectors to prioritize outreach to SSWs. Awareness and educational

252 programs, condom distribution, testing and health check-ups should all be part of a

253 comprehensive strategy for HIV/STI prevention in this high-risk population.

\section{$254 \quad$ List of abbreviations}

255 AIDS: acquired immunodeficiency syndrome

256 BSWs: brothel-based sex workers

257 CDC: Center for Disease Control

258 CNY: Chinese Yuan

259 ELISA: enzyme-linked immunosorbent assay

260 FQ-PCR: real-time fluorescent quantitative polymerase chain reaction 
261 FSWs: female sex workers

262 HIV: human immunodeficiency syndrome

263 NGOs: non-governmental organizations

264 RPR: rapid plasma regain

265 SPSS: Statistical Package for the Social Sciences

266 SSWs: street-based sex workers

267 STI: sexually transmitted infection

268 TPPA: Treponema pallidum particle agglutination

269 USD: United States Dollar

\section{Declarations}

\section{Ethics approval and consent to participate}

272 The research protocol was officially approved by the appropriate sectors of the Chinese

273 government. The field surveys were conducted after obtaining approval from the Ethics

274 Committee for the biomedical ethics review committee of Yunnan, China. Informed

275 consent was obtained from all individual participants included in the study.

\section{Consent for publication}


277 The authors declare that the research was conducted in the absence of any commercial

278 or financial relationships that could be construed as a potential conflict of interest.

\section{Availability of data and materials}

280 Not applicable.

\section{Conflicts of interest}

282 The author reports no conflicts of interest in this work.

\section{Funding}

284 This study was supported by grants from the Japan Society for the Promotion of Science

285 (JSPS KAKENHI Grant Number: JP21790592)

\section{Authors' ccontributions}

287 Cai, Liu (Yufen), and He designed the study. Cai, Liu (Yufen), and Zhang (Jianping)

288 collected the data. Cai, Liu (Yixiao), Hu, Zhang (Jinman), and He analyzed the data. Cai,

289 Liu (Yufen), Liu (Yixiao), Wu, and Zhang (Jinman) drafted the manuscript. He

290 contributed to the interpretation of the results and critical revision of the manuscript for

291 important intellectual content and approved the final version of the manuscript. All

292 authors have read and approved the manuscript. 
295 The authors wish to thank all those who took part in the study and the NGO Bless China

296 International, who helped us to interview participants.

297 Table legends

298 Table 1. Socio-demographic characteristics of street-based and brothel-based FSWs* in

299 Yunnan, China, 2008.

300 Table 2. HIV/STI-related risks and history of sex work of streetbased and brothel based

301 FSWs in Yunnan, China, 2008.

302 Table 3. HIV/STIs healthcare service, knowledge, and risk perception of street-based

303 and brothel-based FSWs* in Yunnan, China, 2008.

304 Table 4. HIV/STIs test results of street-based and brothel-based FSWs* in Yunnan,

305 China, 2008.

306

307

308

309 
311 1. Huang M, Ye L, Liang B, Ning C, Roth W, Jiang J, et al. Characterizing the

312 HIV/AIDS Epidemic in the United States and China. International journal of

313 environmental research and public health. 2015;13(1):ijerph13010030.

314 2. Wu Z, Chen J, Scott S, McGoogan J. History of the HIV Epidemic in China.

315 Current HIV/AIDS reports. 2019;16(6):458-66.

316 3. National Health and Family Planning Commission of the People's Republic of

317 China. 2015 China AIDS Response Progress Report

318 http://wwwunaidsorg/sites/default/files/

319 4. UNAIDS. UNAIDS data 2019.

320 https://wwwunaidsorg/en/resources/documents/2019/2019-UNAIDS-data Accessed 12

$321 \quad$ Feb 2021.

5. Zhou C, Rou K, Dong W, Wang Y, Dong W, Zhou Y, et al. High prevalence of HIV and syphilis and associated factors among low-fee female sex workers in mainland

324 China: a cross-sectional study. BMC infectious diseases. 2014;14:225.

6. Wang L, Wang N, Wang L, Li D, Jia M, Gao X, et al. The 2007 Estimates for People at Risk for and Living With HIV in China: Progress and Challenges. Journal of acquired immune deficiency syndromes (1999). 2009;50(4):414-8. 

2003;289(10):1265-73.

8. M L. China's sex industry flourishing despite dangerous conditions and corrupt

\section{2 police South China Morning Post}

https://wwwmirrorcouk/all-about/south-china-morning- post Accessed 12 Feb 2021.

9. Huang Y, Henderson G, Pan S, Cohen M. HIV/AIDS risk among brothel-based female sex workers in China: assessing the terms, content, and knowledge of sex work.

336 Sexually transmitted diseases. 2004;31(11):695-700. population representative samples of female sex workers in China using Respondent Driven Sampling. Social science \& medicine (1982). 2015;125:79-93. correlates among female sex workers at hair salons: a cross-sectional study in Zhejiang province, China. BMC public health. 2017;17(1):910. 

street-based sex workers (SSWs) in Chongqing, China: interviews with SSWs, clients and healthcare providers. Health \& social care in the community. 2016;24(6):e173-e80. strategies among street-based female sex workers in Chongqing, China. International journal of environmental research and public health. 2015;12(1):855-70. model-based analysis of associations between risk environment and infrequent condom use among Chinese street-based sex workers. PloS one. 2018;13(5):e0195982. STD prevalence between street-based and establishment-based FSWs in Guangdong Province, China. AIDS and behavior. 2012;16(4):943-51. behaviors and syphilis prevalence across types of female sex workers: a preliminary study in Liuzhou, China. Sexually transmitted diseases. 2012;39(3):195-200. and interventions. BMC infectious diseases. 2012;12:84. 
364 19. Strega S, Shumka L, Hallgrímsdóttir H. The "Sociological Equation": Intersections

365 Between Street Sex Workers' Agency and Their Theories about Their Customers.

366 Journal of sex research. 2020:1-12.

367 20. Simon V, Ho. HIV/AIDS epidemiology, pathogenesis, prevention, and treatment.

368 Lancet. 2006;368.

369 21. Wang D, Mei G, Xu X, Zhao R, Ma Y, Chen R, et al. Chinese non-governmental

370 organizations involved in HIV/AIDS prevention and control: Intra-organizational social

371 capital as a new analytical perspective. Bioscience trends. 2016;10(5):418-23.

372 22. Lau J, Wang Z, Kim Y, Li J, Gu J, Mo P, et al. Low sustainability, poor governance,

373 and other challenges encountered by grassroots non-governmental organizations

374 targeting HIV prevention for men who have sex with men in China - a nation-wide

375 study. AIDS care. 2017;29(12):1480-90. 


\section{Supplementary Files}

This is a list of supplementary files associated with this preprint. Click to download.

- Tables.pdf

- Questionnaire.pdf

- supplementarytable.pdf 